\title{
Digital image restoration by Wiener filter in 2D case
}

\author{
A. Khireddine ${ }^{\mathrm{a}, \mathrm{b}, *}, \mathrm{~K}$. Benmahammed ${ }^{\mathrm{b}}$, W. Puech ${ }^{\mathrm{c}}$ \\ ${ }^{a}$ Electronic Department, Faculty of Sciences, University of Bejaia, Algeria \\ ${ }^{\mathrm{b}}$ Electronic Department, Faculty of Sciences, University of Setif, Algeria \\ ${ }^{\mathrm{c}}$ Lirmm Laboratory, University of Montpellier II, France
}

Received 12 October 2004; received in revised form 24 August 2006; accepted 3 October 2006

Available online 15 February 2007

\begin{abstract}
Modern digital technology has made it possible to manipulate multi-dimensional signals with systems that range from simple digital circuits to advanced parallel computers. The goal of this manipulation can be divided into three categories:

- Image Processing image in $\rightarrow$ image out.

- Image Analysis image in $\rightarrow$ measurements out.

- Image Understanding image in $\rightarrow$ high-level description out.

Further, we will restrict ourselves to two-dimensional (2D) image processing although most of the concepts and techniques that are to be described can be extended easily to three or more dimensions. The Wiener filter is a solution to the restoration problem based upon the hypothesized use of a linear filter and the minimum mean-square (or rms) error criterion. In the example given below the image $a[m, n]$ was distorted by a bandpass filter and then white noise was added to achieve an $\mathrm{SNR}=30 \mathrm{~dB}$.
\end{abstract}

(C) 2006 Published by Elsevier Ltd.

Keywords: Digital images; Fourier transform; Sampling; Wiener filter; Noise

\section{Digital image definition}

A digital image $a[m, n]$ described in a $2 \mathrm{D}$ discrete space is derived from an analog image $a(x, y)$ in a $2 \mathrm{D}$ continuous space through a sampling process that is frequently referred to as digitization. The effect of digitization is shown in Fig. 1.

The 2D continuous image $a(x, y)$ is divided into $N$ rows and $M$ columns. The intersection of a row and a column is termed a pixel. The value assigned to the integer coordinates $[m, n]$ with $\{m=0,1,2, \ldots, M-1\}$ and $n=$ $0,1,2, \ldots, N-1\}$ is $a[m, n]$.

We will consider the case of $2 \mathrm{D}$, monochromatic, static images $[1,2]$ :

\footnotetext{
* Corresponding author. Address: Electronic Department, Faculty of Sciences, University of Setif, Algeria.

E-mail addresses: khierben@ieee.org, abkhir@caramail.com(A. Khireddine), william.puech@lirmm.fr (W. Puech).
}

The image shown in Fig. 1 has been divided into $N=16$ rows and $M=16$ columns. The value assigned to every pixel is the average brightness in the pixel rounded to the nearest integer value. The process of representing the amplitude of the 2D signal at a given coordinate as an integer value with $L$ different gray levels is usually referred to as amplitude quantization or simply quantization.

\section{Fourier transform}

The Fourier transform produces another representation of a signal, specifically a representation as a weighted sum of complex exponentials. Because of Euler's formula [5]:

$\mathrm{e}^{\mathrm{j} q}=\cos (q)+\mathrm{j} \sin (q)$

where $\mathrm{j}^{2}=-1$, we can say that the Fourier transform produces a representation of a (2D) signal as a weighted sum of sines and cosines. The defining formulas for the forward 


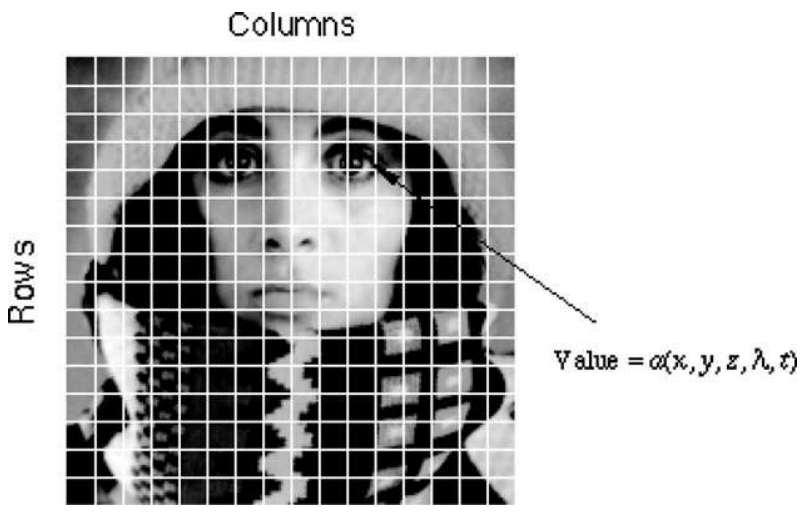

Fig. 1. Digitization of a continuous image. The pixel at coordinates $[m=10, n=3]$ has the integer brightness value 110 .

Fourier and the inverse Fourier transforms are as follows. Given an image $a$ and its Fourier transform $A$, then the forward transform goes from the spatial domain (either continuous or discrete) to the frequency domain which is always continuous $[6,7]$.

\section{Forward:}

$A=F\{a\}$

The inverse Fourier transform goes from the frequency domain back to the spatial domain.

Inverse:

$a=F^{-1}\{A\}$

The Fourier transform is a unique and invertible operation so that

$a=F^{-1}\{F\{a\}\}$ and $A=F\left\{F^{1}\{A\}\right\}$

The specific formulas for transforming back and forth between the spatial domain and the frequency domain are given as

Forward:

$A(u, v)=\int_{-\infty}^{+\infty} \int_{-\infty}^{+\infty} a(x, y) \mathrm{e}^{-\int u x+v y} \mathrm{~d} x \mathrm{~d} y$

Inverse:

$a(x, y)=\frac{1}{4 \pi^{2}} \int_{-\infty}^{+\infty} \int_{-\infty}^{+\infty} A(u, v) \mathrm{e}^{+\int u x+v y} \mathrm{~d} u \mathrm{~d} v$

\section{Spectral sensitivity}

There are several ways to describe the sensitivity of the human visual system.

The perceived intensity as a function of $\lambda$, the spectral sensitivity, for the "typical observer" is shown in Fig. 2. The high sensitivity of silicon in the infra-red means that, for applications where a CCD (or other silicon-based) camera is to be used as a source of images for digital image processing and analysis, consideration should be given to using an IR blocking filter [8,9].

Sensors, such as those found in cameras and film, are not equally sensitive to all wavelengths of light.

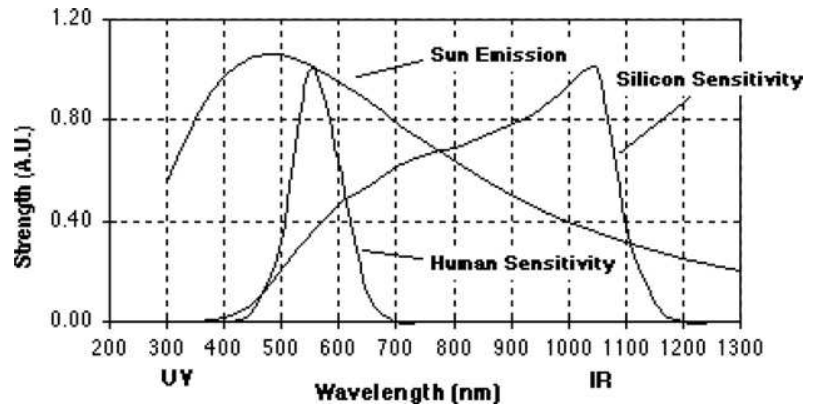

Fig. 2. Spectral characteristics of silicon, the sun, and the human visual system. $\mathrm{UV}=$ ultraviolet and IR $=$ infra-red.

\section{Summary of smoothing algorithms}

A variety of smoothing filters have been developed that are not linear. While they cannot, in general, be submitted to Fourier analysis, their properties and domains of application have been studied extensively. We have:

\subsection{Median filter}

A median filter is based upon moving a window over an image (as in a convolution) and computing the output pixel as the median value of the brightnesses within the input window. If the window is $J * K$ in size we can order the $J * K$ pixels in brightness value from smallest to largest. If $J * K$ is odd then the median will be the $J * K+1 / 2$ entry in the list of ordered brightnesses.

\subsection{Kuwahara filter}

Edges play an important role in our perception of images as well as in the analysis of images. Although this filter can be implemented for a variety of different window shapes, the algorithm will be described for a square window of size $J=K=4 L+1$ where $L$ is an integer. The window is partitioned into four regions as shown in Fig. 3.

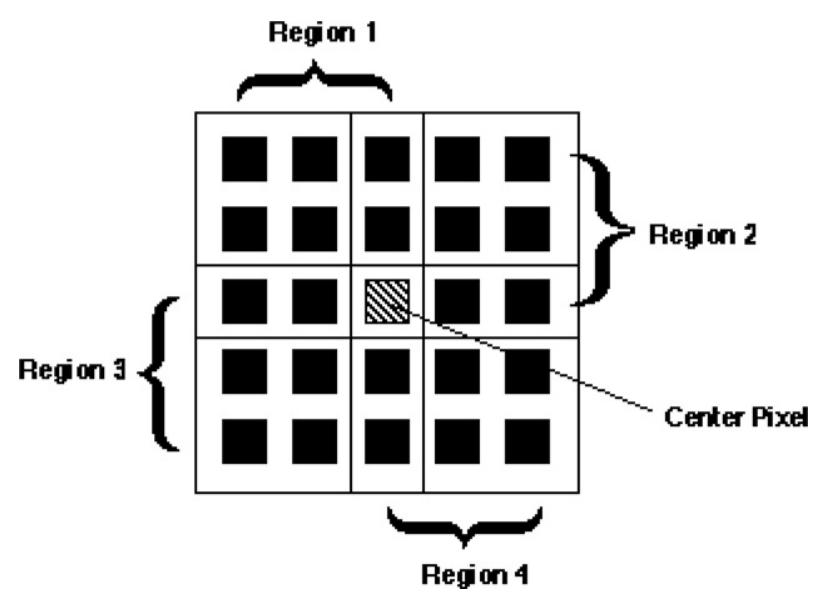

Fig. 3. Four, square regions defined for the Kuwahara filter. In this example $L=1$ and thus $J=K=5$. Each region is $[(J+1) / 2] \times[(K+1) / 2]$. 
Table 1

Characteristics of smoothing filters

\begin{tabular}{llllll}
\hline Algorithm & Domain & Type & Support & Separable/Incremental & Complexity/Pixel \\
\hline Uniform & Space & Linear & Square & Y/Y & $O($ constant $)$ \\
Uniform & Space & Linear & Circular & N/Y & $O(K)$ \\
Triangle & Space & Linear & Square & Y/N & $O($ constant $)$ \\
Triangle & Space & Linear & Circular & N/N & $O(K)$ \\
Gaussian & Space & Linear & $\infty$ & Y/N & $O($ constant $)$ \\
Median & Space & Non-linear & Square & N/Y & $O(K)$ \\
Kuwahara & Space & Non-linear & Square & N/N & $O(J * K)$ \\
Other & Frequency & Linear & - & $-/-$ & $O(\log N)$ \\
\hline
\end{tabular}

a

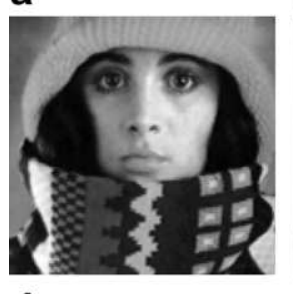

d

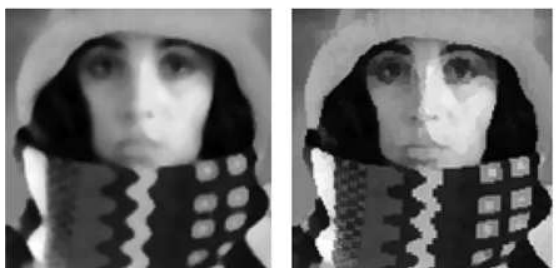

Fig. 4. Illustration of various linear and non-linear smoothing filters. (a) Original, (b) uniform $5 \times 5$, (c) Gaussian $(\sigma=25)$, (d) median $5 \times 5$, (e) Kuwahara $5 \times 5$.

Table 1 summarizes the various properties of the smoothing algorithms. The filter size is assumed to be bounded by a rectangle of $J * K$ where, without loss of generality, $J \geqslant K$. The image size is $N \times N$.

Examples of the effect of various smoothing algorithms are shown in Fig. 4.

\section{Noise suppression}

The techniques available to suppress noise can be divided into those techniques that are based on temporal information and those that are based on spatial information. By temporal information we mean that a sequence of images $\left\{a_{p}[m, n] \mid p=1,2, \ldots, P\right\}$ are available that contain exactly the same objects and that differ only in the sense of independent noise realizations. If this is the case and if the noise is additive, then simple averaging of the sequence $[10,11]$ :

Temporal averaging:

$\hat{a}[m, n]=\frac{1}{p} \sum_{p-1}^{p} a_{p}[m, n]$

\section{Results}

Within the class of linear filters, the optimal filter for restoration in the presence of noise is given by the Wiener filter. The word "optimal" is used here in the sense of minimum mean-square error (mse). Because the square root operation is monotonic increasing, the optimal filter also minimizes the root mean-square error (rms). The Wiener filter is characterized in the Fourier domain and for additive noise that is independent of the signal it is given by [12]

$H_{W}(u, v)=\frac{S_{a a}(u, v)}{S_{a a}(u, v)+S_{n n}(u, v)}$

where $S_{a a}(u, v)$ is the power spectral density of an ensemble of random images $\{a[m, n]\}$ and $S_{n n}(u, v)$ is the power spectral density of the random noise. If we have a single image then $S_{a a}(u, v)=|A(u, v)|^{2}$.

If a two-dimensional signal $a(x, y)$ has Fourier spectrum $A(u, v)$ then

$A(u=0, v=0)=\int_{-\infty}^{+\infty} \int_{-\infty}^{+\infty} a(x, y) \mathrm{d} x \mathrm{~d} y$ a

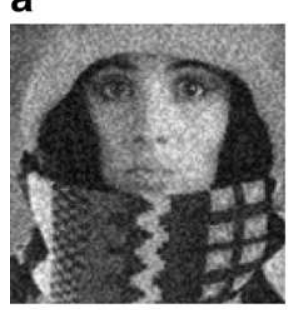

d

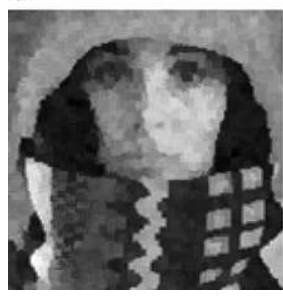

b

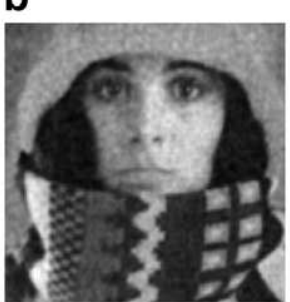

e

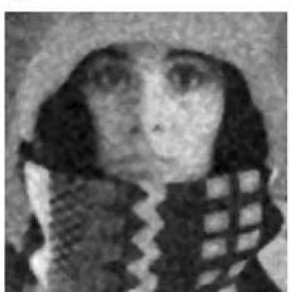

C

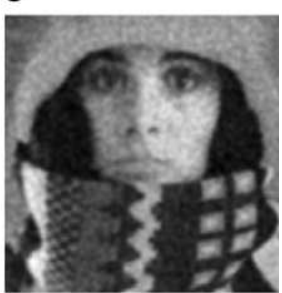

f

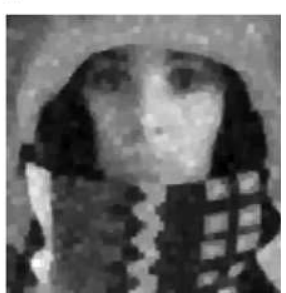

Fig. 5. Noise suppression using various filtering techniques. (a) Noisy image $(\mathrm{SNR}=20 \mathrm{~dB})$, (b) Wiener filter, (c) Gauss filter $(\sigma=1.0)$ $\mathrm{rms}=25.7, \mathrm{rms}=20.2, \mathrm{rms}=21.1$, (d) Kuwahara $(5 \times 5)$, (e) median filter $(3 \times 3)$, (f) Morph. smoothing $(3 \times 3) \mathrm{rms}=22.4 \mathrm{rms}=22.6 \mathrm{rms}=26.2$. 
$a(x=0, y=0)=\frac{1}{4 \pi^{2}} \int_{-\infty}^{+\infty} \int_{-\infty}^{+\infty} A(u, v) \mathrm{d} x \mathrm{~d} y$

The Wiener filter was constructed directly from Eq. (8), because the image spectrum and the noise spectrum were known. The parameters for the other filters were determined choosing that value (either or window size) that led to the minimum rms [13].

\section{Conclusion}

The root mean-square errors (rms) associated with the various filters are shown in Fig. 5. For this specific comparison, the Wiener filter generates a lower error than any of the other procedures that are examined here. The two linear procedures, Wiener filtering and Gaussian filtering, performed slightly better than the three non-linear alternatives.

\section{References}

[1] Khireddine A, Benmahammed K. Application of intelligent matching to automatic speech recognition. IEE(ISH) 1999;II-5:2.67.P6-70.P6.

[2] Gharieb RR. Cumulant-based LP method for two-dimensional spectral estimation. VISP 1999;146(6):307.
[5] Khan IR, Ohba R, Hozumi N. Mathematical proof of explicit formulas for tap-coefficients of full-band FIR digital differentiators. VISP 2000;147(6):553-6.

[6] Towghi N, Pan L, Javidi B. Noise robustness of nonlinear filters for image recognition. JOSA-A 2001;18(9):2054-71.

[7] Holmes AS, Rose CJ, Taylor CJ. Transforming pixel signatures into an improved metric space. IVC 2002;20(9-10):701-7.

[8] Lee DS, Kim GY, Choi HI. A Web-based collaborative filtering system. PR 2003;36(2):519-26.

[9] Leonardis A, Bischof H. Kernel and subspace methods for computer vision. PR 2003;36(9):1925-7.

[10] Gao H, Wang C. Robust energy-to-peak filtering with improved LMI representations. VISP 2003;150(2):82-9.

[11] Rajagopal R, Potter LC. Multivariate MIMO FIR inverses. IP 2003 12(4):458-65.

[12] Pei SC, Tseng CC. An efficient design of a variable fractional delay filter using a first-order differentiator. SPLetters 2003;10(10):307-10.

[13] Bennett M. Fast algorithm for detecting a small signal in the presence of normally distributed noise. VISP 2004;151(4):264-70.

\section{Further reading}

[3] Kumar BVKV, Mahalanobis A, Takessian A. Optimal tradeoff circular harmonic function correlation filter methods providing controlled in-plane rotation response. IP 2000;9(6):1025-34.

[4] Cherniakov M, Sisov VI, Donskoi L. Synthesis of a periodically timevarying digital filter. VISP 2000;147(5):393-9. 